\title{
Distributed Cognition for Evaluating Healthcare Technology
}

\author{
Atish Rajkomar \\ UCLIC \\ University College London \\ Gower Street \\ London, WC1E 6BT, UK \\ atish.rajkomar.09@ucl.ac.uk
}

\author{
Ann Blandford \\ UCLIC \\ University College London \\ Gower Street \\ London, WC1E 6BT, UK \\ a.blandford@ucl.ac.uk
}

\begin{abstract}
Distributed Cognition (DCog) has been proposed as being a better approach to analyzing healthcare work than traditional cognitive approaches, due to the collaborative nature of healthcare work. This study sought to explore this by applying two DCog frameworks, DiCoT and the Resources Model, to the analysis of infusion pump use in an Intensive Care Unit. Data was gathered through observations and interviews, and then analysed using DiCoT and the Resources Model to construct models representing the social structures, information flows, physical layouts and artefact use involved in infusion administration in the ICU. The findings of the study confirm that DCog can be a methodology of choice for studying healthcare work: nurses collaborated significantly, artefacts played a major role in coordinating activity, and the physical environment influenced activity - properties which DCog effectively supports reasoning about.
\end{abstract}

Infusion Pump; Intensive Care Unit; Distributed Cognition; DiCoT; Resources Model.

\section{INTRODUCTION}

There is a need to understand how health practitioners use infusion pumps in context, to improve the design of the devices; incidents occasionally occur during infusion administration, compromising patient safety or even causing death, in general wards (Husch et al., 2005; Vicente, Kada-Bekhaled, Hillel, Cassano, \& Orser, 2003; Taxis \& Barber, 2003), and also in the Intensive Care Unit (ICU) specifically (Malhotra, Laxmisan, Keselman, Zhang, \& Patel, 2005; Shulman, Singer, Goldstone, \& Bellingan, 2005).

Researchers have called for taking a Distributed Cognition (DCog) approach when analyzing human-computer interaction in the healthcare context (Hazlehurst, Gorman, \& McMullen, 2008; Nemeth, 2006; Nemeth, Nunnally, O'Connor, Klock, \& Cook, 2005; Patel \& Kushniruk, 1998; Patel, Cytryn, Shortliffe, \& Safran, 2000). Essentially, the classical model of individual cognition tends to be limiting, because the demarcation of roles and responsibilities of personnel within the team are blurred in practice, and it mixes up the processing performed by individuals with the processing performed by the larger systems in which work is carried out. The question addressed in this research is whether or not DCog supports reasoning about the design and use of one type of device (infusion pumps) in a hospital, taking the ICU as an example, and what roles Distributed Cognition for Teamworking systems (DiCoT) (Furniss \& Blandford, 2006) and the Distributed Information Resources Model (Resources Model) (Wright, Fields, \& Harrison, 2000) can play in supporting analysis. It also delivers an improved understanding on the situated use of infusion pumps, based on this analysis.

Malhotra et al. (2005) describe the ICU as a unique and dynamic setting where multiple individuals provide physical and emotional support to critically ill patients. They note that, to an outsider, the workflow is difficult to understand and may seem very disorganized, especially with the beeping alarms and flashing displays of medical equipment. The high-risk patients admitted have many medical complications that require intensive monitoring, interventions, and a range of medications to stabilize them. Consulting physicians, attending physicians, and nurses, each possessing specialized and sometimes overlapping knowledge, are all involved in decision making around patient care. In the seemingly chaotic environment, the team works together in a coordinated way and relies on sophisticated patient care technology, including infusion pumps.

\section{STUDIES OF INFUSION PUMPS}

To understand how infusion pumps are really used in practice, situated studies are required, to look at 
the messy practical details of work, since clinicians typically spend more of their cognitive resources dealing with these details than with the medical aspects of problems (Nemeth, Cook, \& Wears, 2007). One should search for workplace conflicts, complexities and uncertainties, and investigate how people cope with them, with the aim of exposing strategies and judgements, so that their robustness can be assessed. Hutchins (1995) also states that the cognitive elements of work can be discovered only empirically by the study of work in its natural setting. However, very few field studies have been done due to ethical and privacy issues, and due to efforts by institutions to protect clinicians from scrutiny by "outsiders" (Nemeth et al., 2005).

Studies done on the use of infusion pumps in the ICU so far have focused on identifying sources of errors and quantifying their occurrences (Shulman et al., 2005; Wright, 1991), and on evaluating pump interfaces using techniques such as Heuristic Evaluation (Graham et al., 2004). The only study found that focussed on qualitatively understanding how people use the devices in context was the observational study of Carayon et al. (2005). They studied how nurses use three different types of infusion devices in different hospital units, including the ICU. The observation method gave them insight into the tasks actually carried out versus prescribed tasks, and they identified divergences in practice that were considered to increase risks of failure.

\section{DISTRIBUTED COGNITION}

DCog is distinguished by two related theoretical principles (Hollan, Hutchins, \& Kirsh, 2000). The first principle, pertaining to the boundaries of the unit of analysis for cognition, stipulates that cognitive processes should be looked for, irrespective of physical location, on the basis of the functional relationships of elements that participate in the process. Traditional views of cognition, on the other hand, consider the boundaries to be those of individuals. According to DCog, a system can reorganize itself to bring subsystems into coordination to achieve different functions.

The second principle, concerning the mechanisms that take part in cognitive processes, states that a larger class of events should be looked for, such as the manipulation of external objects and the flow of information representations among actors, on top of looking at the manipulation of symbols inside individual actors. Besides providing extra memory to the same processes that operate on internal memories, the physical environment presents opportunities to reconfigure the distributed cognitive system to take advantage of different combinations of internal and external processes.
When these principles are applied to the observation of human activity, three kinds of distribution of cognition are seen: distribution across the members of a social group, distribution among internal and external (material or environmental) structure, and distribution through time such that the results of earlier events transform later events. Hollan et al. (2000) state that, to understand human cognitive potential, and to design effective human-computer interactions, it is essential to grasp the nature of these distributions of process. Building on these principles and their study of emergency medical dispatch, Furniss and Blandford (2006) developed DiCoT, a codified method for applying distributed cognition to the analysis of sociotechnical systems.

\subsection{DiCoT}

DiCoT focuses on building models to capture the information flows, physical layouts and artefacts of systems. A High Level Input-Output model describes the overall goal of the system in terms of its input, system factors determining the processing, and output. An Information Flow Model describes the information flows among the actors of the system in terms of the communication channels used and key flow properties such as formal versus informal communication, information transformation, information filtering, information buffering, and decision hubs. A Physical Layout Model analyses how physical structures at different levels support communication among actors and facilitate access to artefacts. It also looks at how spatial arrangement supports cognition, based on principles such as perception, naturalness, bodily supports, horizon of observation and situation awareness. An Artefact Model analyses how the detailed design, structure and use of artefacts aid actors in their cognitive work.

The framework has been applied successfully to analyse safety critical systems such as emergency medical dispatch (Furniss \& Blandford, 2006), mobile healthcare work (McKnight \& Doherty, 2008), and underground line control (Webb, 2008). Webb extended DiCoT with two additional models: a Social Structures Model that examines how cognition is socially distributed within the system by looking at the mapping between social structures and goal structures, the sharing of work, and the development and retention of knowledge; a System Evolution Model looks at the evolution of the system over time to understand why work is arranged in a particular way. In this study, data was gathered to build these models for the context of infusion administration in the ICU. In addition, it was found that surrounding system activity was a major influence on infusion pump use, so a new System Activity model was developed to capture these influences. Finally, since the infusion pump 
was a key focus, the Artefact Model described above was extended with a resource-oriented view of the task of administering an infusion, with the aid of the Distributed Information Resources Model, or Resources Model (Wright et al., 2000).

\subsection{The Resources Model}

The Resources Model (Wright, et al., 2000) provides a way to analyse the interactions between actors and artefacts, in terms of the information resources utilised. It differentiates between an abstract information structure and its representation (implementation) in an interaction, and supports the analysis of how an information structure is distributed between actors and artefacts. Abstract information structures can be plans, goals, system states, possibilities, histories, or action-effect relations. Based on the configuration of information resources, different interaction strategies are possible: plan following, plan construction, goal matching, and history-based elimination/selection.

\subsection{DCog and Healthcare}

DCog has already been applied in healthcare to understand the role of cognitive artefacts (Nemeth, 2006; Nemeth, Nunnally, O'Connor, \& Cook, 2006; Xiao, 2005), to identify bottlenecks that can lead to errors in a psychiatric emergency department (Cohen, Blatter, Almeida, Shortliffe, \& Patel, 2006), and to analyse mobile health care work (McKnight \& Doherty, 2008). Regarding infusion pumps, Obradovich and Woods (1996) studied the use of the devices by nurses and patients in the home using a DCog approach. They identified classic $\mathrm{HCl}$ deficiencies in the pump, and strategies adopted by nurses to protect themselves from failure.

\section{METHOD}

Data was gathered over a period of 4 weeks; a total of 20 hours were spent in the ICU. The data gathering process comprised observing nurses at work, general observations of activities and physical layouts, and interviews with bay nurses, nurses-in-charge, senior educator nurses, a medical physicist, a doctor, a healthcare assistant and a cleaner. Structured notes were taken for both observations and interviews, as video recording was not possible due to privacy reasons, and audio recording was not practical due to the ad-hoc and sporadic nature of interviews. A list of data gathering points was prepared, based on the principles of DiCoT and the Resources Model, to guide the observations and interviews. The collected data was analysed using the lens of DCog, by creating representations in the form of diagrams and narratives for each DiCoT model. For all models, the initial representations guided further data gathering efforts, which refined them.

\section{RESULTS}

The results of the study are presented in the form of the different models of DiCoT, as outlined above, highlighting the kinds of insights that each model delivered. The ICU consists of 5 bays, a bay being a cluster of beds in a separate room, with a total of 36 beds. Each bed has an infusion pump station next to it, and has one or more nurses assigned.

\subsection{High Level Input-Output Model}

The overall function of the ICU is to receive patients from other wards who are in a critical state and are in need of intensive monitoring, or who need post-anaesthetic care, and to take care of them until their condition is stable enough for them to be returned to normal wards. Resources in the form of medical staff and medical equipment are allocated to patients depending on the level of care required and on the availability of resources, with the aim of maximising the quality and safety of patient care. This model simply gives a broad overview of the processes and resources involved.

\subsection{System Activity Model}

The next model elaborates on the activity of infusion administration and describes other activities that potentially influence it. This model is new; the need for it emerged from the complexity and dynamism of the ICU environment. Many observed phenomena belonged to "secondary" activities, which were independent of infusion administration, the "primary" activity for this study, but which contributed to achieving the overall system goal of providing patient care. These secondary activities could influence infusion administration by, for example, changing the physical environment or causing interruptions, and therefore should be considered in the analysis. The System Activity Model was developed to describe all these activities, first at a high level in the form of a consolidated diagram, see Figure 1, and then at a lower level in the form of a summary for each activity. For the primary activity, another level of detail was added to describe its different tasks.

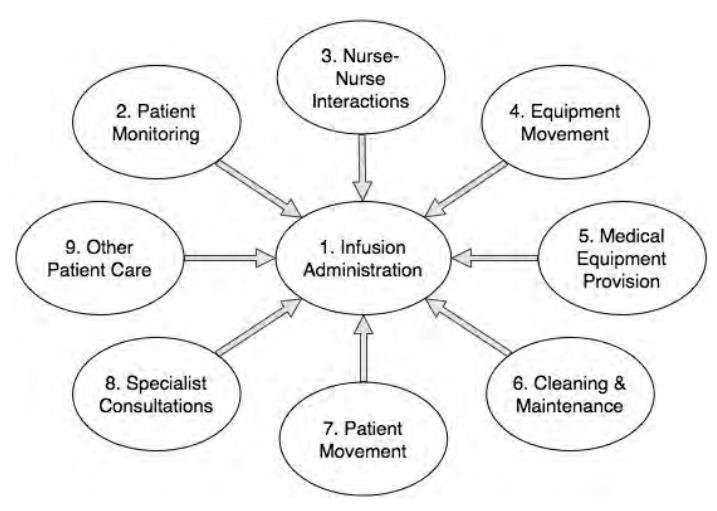

Figure 1: System Activity Model of the ICU 


\subsubsection{Infusion Administration}

The primary activity is infusion administration. The doctor examines the new ICU patient's file to decide which drugs need to be prescribed, based on inputs from the surgeon and the anaesthetist. The doctor then enters all prescriptions into the Electronic Patient Record (EPR). The nurse prepares and administers the required intravenous drugs to the patient. This consists of the following sub-tasks:

(i) Reading the prescriptions from the EPR.

(ii) Calculating the required medication dose and flow rate based on medication information available in the Drug Chart menu of the EPR, taking into consideration patient-specific parameters such as body weight.

(iii) Preparing the medication at the required concentration, putting it into the syringe/bag (syringe in case of a syringe driver pump or bag in case of a volumetric pump) and priming the syringe/bag. Priming means pushing the liquid to the tip of the syringe/bag so that all air bubbles are removed.

(iv) Connecting the syringe/bag to the infusion pump and to the patient.

(v) Programming the infusion rate (and optionally volume to be infused).

(vi) Starting the infusion.

Thereafter, the nurse replaces the syringe/bag when required, to ensure continuous delivery of the drug. The replacement is usually prepared in advance for a smooth transition. The nurse also checks the drug volume infused every hour and inputs it to the EPR. If the nurse is concerned about the patient's state, he/she contacts the doctor, who examines the patient again. If required, the doctor updates the prescriptions and the nurse changes the infusions accordingly.

\subsubsection{Secondary Activities}

Eight secondary activities were identified as having an influence on the primary activity, as follows.

Patient Monitoring: The nurse regularly checks the patient's condition physically and on the life support system. This activity could be interleaved with Infusion Administration, resulting in a multitasking scenario. Also, infusions may need to be modified after certain changes in the patient's condition. For example, when trying to control a patient's blood pressure with an adrenaline infusion, the nurse needs to continuously check the blood pressure and modify the infusion until the desired balance is reached. Lastly, if the life support system alarm goes off while the nurse is preparing an infusion, this will be an interruption.
Nurse-Nurse Interactions: Nurses continuously interact with each other for various reasons, and collaborate significantly. While administering an infusion, a nurse could get interrupted by: another nurse asking for help with something extremely urgent, a senior nurse asking him/her to sign a drug administration sheet, or other nurses stopping by for a friendly chat. Also, the quality of the shift handover determines how well-informed the new shift nurse initially is about the state of ongoing infusions.

Equipment Movement: Medical equipment is continuously moved around in the ICU. It is common for nurses to take idle pumps from other beds, if they cannot easily get a new one from the medical physics stock. This activity influences Infusion Administration by causing changes in the physical layout of the bed area when pumps are displaced.

Medical Equipment Provision: Nurses take defective pumps to the medical physics office, and retrieve spare pumps. According to nurses, sometimes they cannot get a replacement from there, and look for spare pumps at other beds. If a pump breaks down while a nurse is using it, it can be a disruptive interruption to Infusion Administration. Then, the ease with which the nurse can find a replacement pump determines how quickly the infusion can be resumed.

Cleaning \& Maintenance: The ICU has very strict hygiene requirements, and the beds and bays are cleaned regularly throughout the day. Cleaners often move artefacts around, causing changes in the physical layout. Consequently, a nurse might need to reconfigure the physical setup before starting infusions.

Patient Movement: Patients typically stay in the ICU for one night, resulting in high daily patient traffic. Infusion pumps follow patients to other wards if infusions are still required. The displacement of pumps means that the physical layout of the bed area changes.

Specialist Consultations: Specialists, such as microbiologists and physiotherapists, visit the ICU regularly to check patients or to respond to consultation requests. These consultations can result in communications that change infusion requirements.

Other Patient Care: Patient care also consists of other activities such as the provision of meals. These can change the physical layout or serve as distractions. For example, a food trolley could hinder a nurse and block line of sight to infusion attachments. 
This model gives a structured overview of the many influences that can disturb the activity of infusion administration and the broader use of pumps.

\subsection{Social Structures Model}

The Social Structures Model, from the DiCoT adaptation by Webb (2008), aims to understand how the social structures of the organisation map onto the goal structures of the system, how robustness is achieved, and how the system learns through the developing knowledge of the actors.

\subsubsection{Mapping social structure to goal structure}

A hierarchical structure can map to a goal structure such that superordinate and subordinate share responsibility to ensure that sub-goals of the overall goal are satisfied. The diagram in Figure 2 was developed to represent the sharing of goals for infusion administration across the social hierarchy.

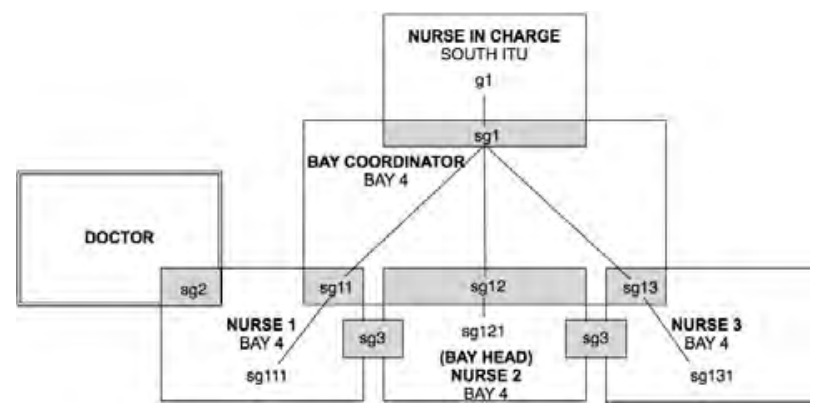

Figure 2. Goal structure of the ICU. Note that $s g 3$ is shared among all nurses.

The goal of the Nurse-in-Charge, g1, is to provide nursing care to all patients in all of the bays of her division. The Nurse-in-Charge and the Bay Coordinator share the sub-goal sg1 of providing nursing care to all patients in a particular bay. The Bay Coordinator is responsible for all patients in a particular bay, and shares the sub-goal sg11 with a Nurse of providing nursing care for a particular bed in the bay.

The Doctor and the Nurse share the sub-goal sg2 of treating the patient with the required drugs and sustaining the patient with life support mechanisms. To treat the patient, a Nurse has individual subgoals, e.g sg111, pertaining to activities defined in the System Activity Model, such as Infusion Administration and Patient Monitoring. During the daily shift handover, a nurse is briefed firstly on his/her particular patient, secondly on all patients in his/her bay, and thirdly on all "hot" (extremely critical) patients across the ICU. When a particular patient's assigned nurse is away, or in case of emergency, another nurse could intervene on the patient's infusions. This can be viewed as another sub-goal sg3 shared among all nurses in the bay.
Nurses collaborate significantly and share work. A highly critical patient can have three nurses assigned and working in parallel, with one nurse setting up infusion pumps, another one checking the life support system, and another one updating the patient's record. Admitting a new patient can require extra manpower, in which case nurses request help from colleagues.

\subsubsection{Achieving system robustness}

The social structures existing in the ICU help make the system more robust by propagating information, creating redundancies, and facilitating error prevention. Information is propagated when nurses update each other on the current states of their patients. This information exchange helps nurses possess up to date knowledge about neighbouring patients, allowing them to intervene on their infusions if required, and, coupled with the shift handover described above, results in a redundancy mechanism. Errors are prevented when, while collaborating or interacting socially, nurses share expertise, advise their colleagues, and doublecheck the task operations of their colleagues.

\subsubsection{Development and retention of knowledge}

The third section of this model focuses on how knowledge is developed, retained and shared. A few senior educator nurses have in-depth knowledge of the functionalities of the pumps and associated procedures, and train new nurses on infusion pump use. The nurse rota is planned such that there is a mixture of experience levels in each bay, allowing junior nurses to learn from senior ones. Important information, such as a new intravenous line dressing being put in use, is posted on notice boards in the coffee room: the coffee room serves both as a social area and as an area where system information is shared. Another platform for knowledge exchange is staff meetings; problems faced and solutions for them are shared, allowing the actors of the system, and hence the system itself, to learn. Some nurses indicated that they had been trained only once on the use of the pumps, and would like to know more functionalities. For example, one nurse was not aware that it was possible to start a continuous flow infusion without specifying a "Volume To Be Infused", and a couple of nurses did not know that they could administer bolus amounts on demand without any programming.

The social structures model highlights how social structures and practices support the work around infusion devices, ensuring that knowledge is disseminated and expertise developed. It also identify shortcomings and opportunities for improvement. While the Social Structures model includes consideration of longer term learning and knowledge exchange, there is a need also to understand the detailed, localised information flows 
that most directly influence interaction with the device; this is the focus for the information flow model.

\subsection{Information Flow Model}

The Information Flow model, from the existing DiCoT framework, describes the information flows that exist in the activity of infusion administration in terms of the communication channels among actors and of the key flow properties. The analysis was scoped around the actors who seemed most important for infusion administration: the patient, the nurse and the doctor.

\subsubsection{Communication channels}

The doctor prescribes drugs in the EPR and the nurse refers to it to know which drugs to administer to the patient. While fitting infusion lines, the nurse asks the patient to cooperate with body movements verbally and/or through touch and gestures. Nurses continuously update the EPR, and the doctor can refer to it when required. Nurses communicate with other nurses formally for shift handover, for administrative formalities, or when asking for assistance, through the EPR and face-to-face. During face-to-face informal conversations, nurses often update each other on the states of their patients and propagate information through the system, illustrating the important role that informal communication channels can play.

\subsubsection{Key flow properties}

The doctor acts as an information decision hub, taking information from his own examinations, from other specialists, and from nurses, to decide what drugs should be prescribed for the patient. The nurse also acts as an information decision hub, taking information about the patient's vital signs from the life support system, and drug-specific information from the computer system, to decide on the drug concentrations that are required to stabilise the patient.

Communication among actors and their access to artefacts are influenced by the physical layout of the environment. The next model examines the physical layout of the ICU.

\subsection{Physical Layout Model}

The Physical Layout Model analyses how the layout supports communication among actors and access to artefacts. Hollan, Hutchins, \& Kirsh (2000) state that when space is used well it reduces the time and memory demands of our tasks and increases the reliability of execution. Spatial arrangements can simplify choice, perception, or internal computation. The physical layout of the ICU was analysed firstly at the bay level, secondly at the bed level, and finally at the infusion setup level.
5.5.1 Bay level

The layout of a typical ICU bay is shown in Figure 3.

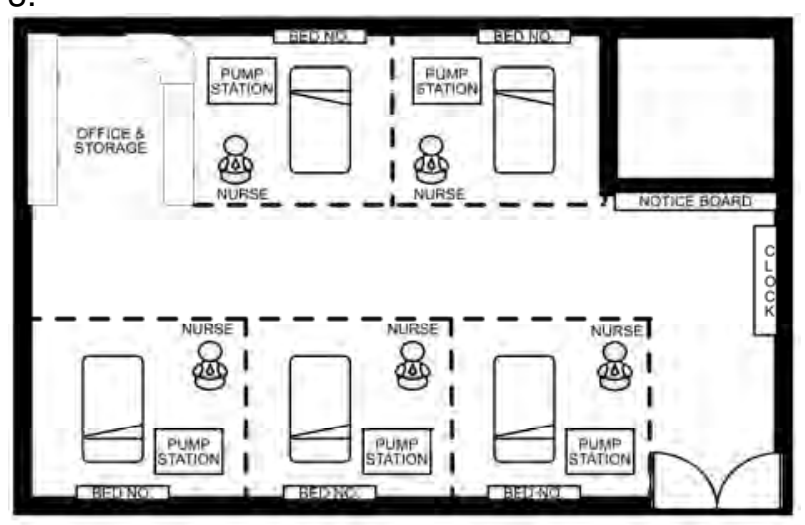

Figure 3. ICU Bay Layout

The dotted lines represent bed curtains, which by default are open, but can be closed when privacy is desired. This open layout allows nurses to interact with each other easily, and allows other staff passing by to communicate with nurses at bedsides. When the curtains are open, nurses can also see patients at opposite beds, providing situation awareness of other patients as well. The office and storage space also serves as an area where nurses and other clinicians congregate for discussions. The notice board is located in the line of sight of incomers. It contains information such as reminders for nurses to label patients' infusion lines with their insertion dates, which is an externalisation of a goal that nurses have while preparing infusions. In essence, the setup facilitates the propagation of information among actors of the system and allows nurses to maintain situation awareness at the level of the bay.

\subsubsection{Bed level}

The layout of an ICU bed is as shown in Figure 4.

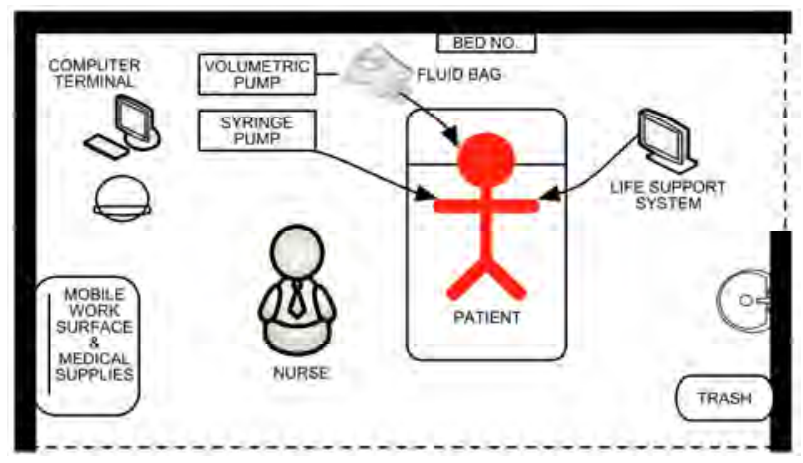

Figure 4. ICU Bed Layout

The layout allows several clinicians to be at the bedside at the same time, when collaborating on problem-solving for example. It also allows a nurse to prepare an infusion, while verbally communicating with the patient and checking the 
life support system, so that the nurse maintains situation awareness despite his/her changing visual horizon of observation. The horizon of observation is the functional workspace that an actor can monitor in addition to performing a specific task.

The main artefacts used in infusion administration are the medical work surface, the computer terminal and the infusion station. A nurse needs to coordinate information resources among these while preparing an infusion, and they are strategically located close together. Additionally, the layout allows all the artefacts to be manned at the same time with, for example, one nurse setting up infusion pumps, another nurse entering data into the EPR, and a third nurse checking the life support readings.

\subsubsection{Infusion setup level}

An infusion station consists of a pump rack with slots for pumps, and an extendable arm with a bag holder. There are typically two to three active pumps, but in some cases there can be up to eight, adding to the complexity of the setup. As noted in section 5.2.2, the number of pumps in a station changes when pumps accompanying an incoming patient are added, or when pumps accompanying an outgoing patient are removed, or when an inactive pump is taken to another bed. The pumps have important physical attributes that support (or fail to support) nurses interacting with them.

Information Externalisation: After preparing a medication at the required concentration, a nurse writes a note with the drug name and concentration, and sticks it onto the container. This is to ensure that in the event of an interruption, or if another nurse takes over the task of administering the infusion, the task can be quickly and correctly resumed. Also, after connecting an infusion line to a patient, a nurse sticks a label onto the tube with the drug name and insertion date, to know when the tube needs to be changed. This externalization into the physical environment ensures that the information is shared with other shift nurses.

Perception versus Cognition: The line connecting a syringe/bag to a patient is colour-coded depending on the type of infusion. For example, epidural anaesthesia lines have orange attachments. This makes the recognition of the type of infusion a perceptual task. When reading off hourly volume intakes from the syringe driver, nurses sometimes need to position themselves awkwardly or displace the syringe, when the volume label and drug label are as shown in Figure 5. The labelling should be such that both labels are easily perceivable.

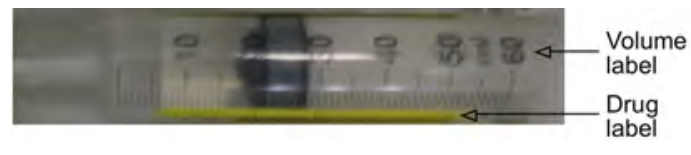

Figure 5. Syringe labels inside pump

Natural Mapping: The order of the pumps in the station does not necessarily match the order in which the drugs are prescribed in the EPR, meaning that whenever recording hourly volume intakes, a nurse has to first check which pump is administering the drug. A natural mapping would make this readily perceivable.

This model looked at how the physical environment of the ICU supports cognition and interaction. The final model analyses how cognition is distributed between an actor and artefacts during the task of administering an infusion.

\subsection{Artefact Model}

The Artefact Model analyses how the design, structure, and use of artefacts support activity. In this study, two types of analysis were done. Firstly, the task of administering a constant flow rate infusion was analysed using the Resources Model to describe the information resources utilised by the nurse, how they are represented through the different artefacts involved, and the implications for the interaction. Secondly, the interface of the pump was analysed to see how the pump, as a specific artefact, supported the activity of infusion administration.

\subsubsection{Distributed information resources analysis}

The Resources Model was applied by mapping the concepts of plan, goal, state, possibilities, history, and action-effects onto state transitions, so that the task of infusion administration could be analysed in terms of information resources tied to state transitions, each representing a discrete step of the task. For each step of the task described in section 5.2.1, the representations of information resources were analysed. The information resources could be represented internally (in the nurse's head) or externally through artefacts such as the infusion pump, the EPR, the syringe/bag, and the medical work surface. The findings of the resource-oriented analysis were that, firstly, besides their normal functions, the various artefacts used in the task served as representations of previously completed goals, that can help a nurse keep track of the current state in the task plan. For example, after a nurse has used the EPR to calculate the required drug concentration, the result of this calculation will be on-screen, serving as a cue that the goal of calculating the concentration has already been achieved. This can help the nurse in state recollection after an interruption. Secondly, throughout the task, the nurse needs to coordinate 
an internal plan representation with internal or external history representations to keep track of the current state in the plan. Having an external representation of the plan could help reduce the cognitive demands on the nurse, especially in the event of interruptions. Thirdly, all the pump control buttons, except the Value-reset / Back button, either have text on the button indicating its purpose, for example the Start / Stop Button has "Start/Stop" on it, or have a commonly used icon on the button, for example the power button sign, from which a user can deduce the purpose of the button. The Value-reset/Back button just has a $\mathrm{C}$ character on it with a circular arrow pointing backwards, and the colour of the button is green, which could be confusing for users, since generally a green colour is used for confirmation / start operations, not for resetting / going back.

\subsubsection{Infusion pump interface analysis}

This part of the analysis focused on the different interactive features of the infusion pump that facilitate or hinder the work of nurses.

Pump Alarms: The pump has a reminder alarm which gets triggered ten minutes before a syringe/bag runs out, to give enough time to prepare an infusion replacement. It reduces the cognitive load of the nurse by externally representing the goal of having to change the syringe soon. The main alarm can be viewed as an information signal that pre-emptively draws the attention of the nurse from other activities to infusion administration.

VTBI Parameter: When starting an infusion, nurses can specify a Volume To Be Infused (VTBI), which acts as a safety limit. Senior educator nurses have discouraged the practice of programming a VTBI for continuous infusions with the syringe driver, following a critical incident: a nurse programmed a volume of $50 \mathrm{ml}$ on a syringe driver pump, changed the syringe after $45 \mathrm{ml}$ had been dispensed, but then forgot to reprogram the pump to run for another $50 \mathrm{ml}$; consequently the pump stopped the delivery after dispensing $5 \mathrm{ml}$ from the new syringe. From a DCog point of view, the pump should externalise and possibly enforce a goal of reprogramming or confirming the VTBI onto the user after a syringe handling operation is detected; the flow should not be resumed until the nurse explicitly confirms the VTBI.

Volume Counter: Every hour, to record the hourly intake of a drug with the volumetric pump, the nurse needs to access a Status menu from the Main Menu of the pump interface, choose an Intermediate Parameters option, read the volume infused, and then reset the counters to zero. The whole operation takes 8 key presses. Nurses find this time-consuming and think that having a button for directly resetting running counters, like one of the previously used pumps had, would improve efficiency.

This model looked at the main artefacts involved in Infusion Administration. The analysis highlighted the importance of artefacts as representations of information resources, and showed the influence of artefact design on work.

\section{DISCUSSION}

\subsection{Assessment of Distributed Cognition}

\subsubsection{Relevance for the healthcare context}

Patel et al. (2000) state that to cope with rising costs of healthcare and to leverage rapid knowledge growth, healthcare professionals are increasingly sharing knowledge and skills. Distributed responsibilities allow the team to process large amounts of information and decrease cognitive load on individuals. The Social Structures Model showed that the social distribution of cognition in the ICU is indeed significant. Firstly, the responsibilities of personnel overlap to ensure that sub-goals of the overall system goal are achieved. Secondly, collaborative problem-solving and sharing of work are inherent in the kind of work performed, and different nurses can intervene on an infusion if required. Thirdly, social interactions are highly encouraged to downplay the mortality and morbidity of the environment, and such interactions influence work. It also revealed that, while senior educator nurses possessed in-depth knowledge of pump functionality, other nurses lacked training in functionalities that could improve task efficiency.

The Physical Layout Model showed that the physical environment of the ICU, at the bay level and at the bed level, influenced: the propagation of information through the system, access to artefacts, and how actors maintain situation awareness of the system. The analysis at the infusion setup level showed how perceptual principles reduce the cognitive work of nurses (colour-coding), and identified improvements in spatial arrangements that could simplify work (position of syringe labels and ordering of pumps). This model also showed that information is externalized into the environment (bay notice board, medication post-it notes, and infusion line date labels), and highlighted how the dynamism of the environment influences work, as pumps are moved around.

The resource-oriented analysis done for the Artefact Model showed that the different artefacts used in infusion administration (infusion pump, EPR, medical supplies) served as representations 
of information resources (goals, states, actioneffects, histories) throughout the infusion administration task, highlighting the crucial role that these artefacts play in supporting and coordinating the task. The analysis of the pump interface showed that the design of the artefact: can reduce cognitive workload (reminder alarms); can contribute to safety incidents (VTBI incident); and can decrease task efficiency (counter-reset). Halverson (2002) argues that by considering artefacts, DCog allows the understanding of the roles of humans versus artefacts, and the assessment of the consequences of automation. The VTBI incident illustrates the importance of this in the healthcare context. Safety is not a property of individual tasks or actions, but of the interrelationships and interconnections between parts of a system (Fields, Paterno, Santoro, \& Tahmassebi, 1999), which makes DCog an attractive methodology for studying safety critical systems. The Information Flow Model stressed the importance of the EPR as an artefact mediating the work of the doctor and the different shift nurses.

The social and external (artefacts and environment) distribution of cognition and the influence of the physical environment on activity that were observed in this study support the claim that DCog is better suited than classical cognition models for analyzing human-computer interactions in a healthcare setting such as the ICU.

\subsubsection{As a methodology for practitioners}

DCog has been criticised for providing little structure for conducting analyses. Furniss and Blandford (2006) attempted to remedy this by developing DiCoT, a codified method for structuring DCog analyses. For this study, the focus and structure that DiCoT provides through its models and their associated principles were found to be effective in supporting the development of understanding. The principles guided the data gathering process by highlighting the types of phenomena to look for.

Hutchins (1995) asserts that applying DCog requires a deep understanding of the work domain being studied. Indeed, one of the major challenges faced while applying DCog to the ICU context were the dependency on observational data and the domain knowledge that needed to be built up, to spot the pertinent details of the work. Building up this domain knowledge can require considerable time; a substantial proportion of observations and interviews for this study were spent on it.

6.1.3. As a theory with the scope to extend analysis For analysing work in the ICU context, the approach used must be able to account for the existence and influences of multiple concurrent activities within the system. DCog theory does not discuss the existence of different activities within a system and how they potentially influence each other. A related point made by Halverson (2002) is that an analyst using DCog has to define a unit of analysis based on the functions of interest, and that the boundaries of this unit could be reshaped as needed. Activity theory (Kaptelinin \& Nardi, 2006) on the other hand, fundamentally defines an activity as the unit of analysis, and Bardram (2009) notes that, using concepts from Activity Theory, a clinician can be described as being involved in more than one activity at a time and being responsible for different actions within each of these activities. Activity Theory may provide more leverage for the System Activity Model than does DCog theory, suggesting that concepts may usefully be drawn from other theories to reinforce distributed cognition analysis for some settings.

\section{CONCLUSION}

The goal of this study was to test the utility and practicality of applying a DCog methodology to the analysis of infusion administration in an Intensive Care Unit. The findings of the study confirm that DCog can be a methodology of choice for studying healthcare work: a high level of collaboration was found among nurses, artefacts were found to play a major role in supporting and coordinating work, and the (dynamic) configuration of the physical environment was found to influence work. These properties imply that an approach which views cognition as being distributed throughout a system, rather than being only in the minds of individuals, is needed to effectively analyse human-computer interactions in such a setting.

\section{ACKNOWLEDGEMENTS}

We are grateful to all participants in this study for the time and expertise they shared with us. This research was partially funded by EPSRC grant EP/G059063/1.

\section{REFERENCES}

Bardram, J. E. (2009) Activity-based computing for medical work in hospitals. ACM Transactions on Computer-Human Interaction, 16(2), 1-36.

Carayon, P., Wetterneck, T. B., Hundt, A. S., Ozkaynak, M., Ram, P., Desilvey, J., et al. (2005) Observing Nurse Interaction with Infusion Pump Technologies. Advances, (4), 349-364.

Cohen, T., Blatter, B., Almeida, C., Shortliffe, E., \& Patel, V. (2006) A cognitive blueprint of collaboration in context: distributed cognition in the psychiatric emergency department. Artificial intelligence in medicine, 37(2), 73-83. 
Fields, R., Paterno, F., Santoro, C., \& Tahmassebi, S. (1999) Comparing design options for allocating communication media in cooperative safety-critical contexts: a method and a case study. ACM Transactions on Computer-Human Interaction (TOCHI), 6(4), 370-398.

Furniss, D., \& Blandford, A. (2006) Understanding emergency medical dispatch in terms of distributed cognition: a case study. Ergonomics, 49(12-13), 1174-203.

Graham, A. A., \& Holohan, T. V. (1994) External and Implantable Infusion Pumps. Technology Assessment report abstract. Agency for Health Care Policy and Research, Rockville, MD.

Graham, M. J., Kubose, T. K., Jordan, D., Zhang, J., Johnson, T. R., Patel, V. L., et al. (2004) Heuristic evaluation of infusion pumps: implications for patient safety in Intensive Care Units. International journal of medical informatics, 73(1112), 771-9.

Halverson, C. A. (2002) Activity Theory and Distributed Cognition: Or What Does CSCW Need to DO with Theories? Computer Supported Cooperative Work (CSCW), 11(1), 243-267.

Hazlehurst, B., Gorman, P. N., \& McMullen, C. K. (2008) Distributed cognition: an alternative model of cognition for medical informatics. International journal of medical informatics, 77(4), 226-34.

Hollan, J., Hutchins, E., \& Kirsh, D. (2000) Distributed cognition: toward a new foundation for human-computer interaction research. ACM Transactions on Computer-Human Interaction (TOCHI), 7(2).

Husch, M., Sullivan, C., Rooney, D., Barnard, C., Fotis, M., Clarke, J., et al. (2005) Insights from the sharp end of intravenous medication errors: implications for infusion pump technology. Quality \& safety in health care, 14(2), 80-6.

Hutchins, E. (1995) Cognition in the wild. MIT Press, Cambridge, MA.

Kaptelinin, V., \& Nardi, B. A. (2006) Acting with technology: activity theory and interaction design. The MIT Press.

Malhotra, S., Laxmisan, A., Keselman, A., Zhang, J., \& Patel, V. L. (2005) Designing the design phase of critical care devices: a cognitive approach. Journal of biomedical informatics, 38(1), 34-50.

McKnight, J., \& Doherty, G. (2008) Distributed cognition and mobile healthcare work. Proceedings of the British $\mathrm{HCl}$ Group Annual Conference, 2008, 2.

Nemeth, C. (2006) Discovering Healthcare Cognition: The Use of Cognitive Artifacts to Reveal Cognitive Work. Organization Studies, 27(7), 10111035.
Nemeth, C. P., Cook, R. I., \& Wears, R. L. (2007) Studying the technical work of emergency care. Annals of emergency medicine, 50(4), 384-6.

Nemeth, C., Nunnally, M., O'Connor, M., \& Cook, R. (2006) Creating resilient IT: how the sign-out sheet shows clinicians make healthcare work. AMIA Annual Symposium Proceedings, 2006, 5848.

Nemeth, C., Nunnally, M., O'Connor, M., Klock, P. A., \& Cook, R. (2005). Getting to the point: developing IT for the sharp end of healthcare. Journal of biomedical informatics, 38(1), 18-25.

Nunnally, M., Nemeth, C. P., Brunetti, V., \& Cook, R. I. (2004) Lost in menuspace: user interactions with complex medical devices. IEEE Transactions on Systems, Man, and Cybernetics Part A: Systems and Humans, 34(6).

Obradovich, J., \& Woods, D. (1996) Users as designers: how people cope with poor $\mathrm{HCl}$ design in computer-based medical devices. Human Factors, 38(4), 574-92.

Patel, V. L., \& Kushniruk, A. W. (1998) Interface design for health care environments: the role of cognitive science. AMIA Annual Symposium Proceedings, 1998, 29-37.

Patel, V. L., Cytryn, K. N., Shortliffe, E. H., \& Safran, C. (2000) The collaborative health care team: the role of individual and group expertise. Teaching and learning in medicine, 12(3), 117-32.

Shulman, R., Singer, M., Goldstone, J., \& Bellingan, G. (2005) Medication errors: a prospective cohort study of hand-written and computerised physician order entry in the intensive care unit. Critical care (London, England), 9(5), R516-21.

Taxis, K., \& Barber, N. (2003) Ethnographic study of incidence and severity of intravenous drug errors. BMJ (Clinical research ed.), 326(7391), 684. Vicente, K. J., Kada-Bekhaled, K., Hillel, G., Cassano, A., \& Orser, B. A. (2003) Programming errors contribute to death from patient-controlled analgesia: case report and estimate of probability. Canadian Journal of Anesthesia/Journal canadien d'anesthésie, 50(4), 328-332.

Webb, P. (2008) Extending a Distributed Cognition Framework: The Evolution and Social Organisation of Line Control. Unpublished Master's Thesis, University College London, London.

Wright, D. (1991) Critical incidents in the intensive therapy unit. The Lancet, 338(8768), 676-678.

Wright, P. C., Fields, R. E., \& Harrison, M. D. (2000) Analyzing human-computer interaction as distributed cognition: the resources model. HumanComputer Interaction, 15(1).

Xiao, Y. (2005) Artifacts and collaborative work in healthcare: methodological, theoretical, and technological implications of the tangible. Journal of biomedical informatics, 38(1), 26-33. 